\title{
An algorithm for calculating the Bethe logarithm and the adiabatic correction for atoms and two-center molecules
}

\author{
Ewa Palikot* \\ Nicolaus Copernicus University in Torun, Poland \\ E-mail: epedoktorant.umk.pl

\section{Monika Stanke} \\ Nicolaus Copernicus University in Torun, Poland \\ E-mail: monika@izyka.umk.pl
}

\begin{abstract}
Bethe logarithm $\ln k_{0}$ is one of the leading quantum-electrodynamics energy corrections of the order of $\alpha^{3}$. A method for calculating this correction, which is an alternative to the method of Schwartz et al. [1], is presented. An effective algorithm for selecting a basis set to be used in the $\ln k_{0}$ calculation is also shown. This work is based on the previous paper by Stanke et al. [2]. The approach proposed by Stanke et al. in that paper is extended for calculating multi-electron atoms and two-center molecules. The proposed algorithm employs a spectral representation of a function of an operator and its spectral decomposition. The calculations are done within the BornOppenheimer approximation using explicitly correlated Gaussian functions with shifted centers. In both cases, the atomic and the molecular, one of the main problems is the choice of appropriate basis sets.
\end{abstract}

International Conference on Precision Physics and Fundamental Physical Constants - FFK2019 9-14 June, 2019

Tihany, Hungary

${ }^{*}$ Speaker. 


\section{Background}

The most convenient theoretical method for calculating bound states of light atoms and molecules is the expansion of the total energy of the system in powers of the fine-structure constant $\alpha$ :

$$
\mathrm{E}=\mathrm{E}^{(0)}+\alpha^{2} \mathrm{E}^{(2)}+\alpha^{3} \mathrm{E}^{(3)}+\alpha^{4} \mathrm{E}^{(4)}+\ldots
$$

where

- $\mathrm{E}^{(0)}$ is the nonrelativistic energy (a solution of the Schrödinger equation),

- $\alpha^{2} \mathrm{E}^{(2)}$ is the expectation value of the Breit-Pauli Hamiltonian, and

- $\alpha^{3} \mathrm{E}^{(3)}, \alpha^{4} \mathrm{E}^{(4)}, \cdots$ are QED corrections.

The QED energy correction proportional to the third power of $\alpha$ is given by:

$$
\begin{aligned}
\mathrm{E}_{3}= & \frac{4}{3} \frac{Z}{m^{2}} \sum_{i}\left[\ln \alpha^{-2}+\frac{19}{30}-\ln k_{0}\right]\left\langle\delta\left(\mathbf{r}_{i}\right)\right\rangle+ \\
& +\sum_{i>j} \sum_{j}\left[\left(\frac{164}{15}+\frac{14}{3} \ln \alpha\right) \frac{1}{m^{2}}\left\langle\boldsymbol{\delta}\left(\mathbf{r}_{i j}\right)\right\rangle-\frac{14}{3} \frac{m}{4 \pi}\left\langle P\left(\frac{1}{r_{i j}^{3}}\right)\right\rangle\right],
\end{aligned}
$$

where

- $\ln k_{0}$ is the Bethe logarithm,

- $P\left(r^{-3}\right)$ is a distribution (so-called Araki-Sucher correction), And

- $\alpha$ is the fine-structure constant.

\section{Method}

The approach developed in this work is based on the general method for calculating matrix elements of an analytic function, $f(\mathbf{A})$, of operator $\mathbf{A}$.

Bethe logarithm is defined as:

$$
\ln k_{0}=\frac{\left\langle\Psi\left|\mathbf{p}\left[\left(\mathrm{H}_{0}-\mathrm{E}_{0}\right) \ln \left(2\left(\mathrm{H}_{0}-\mathrm{E}_{0}\right)\right)\right] \mathbf{p}\right| \Psi\right\rangle}{\frac{1}{2}\left\langle\Psi\left|\left[\mathbf{p},\left[\mathrm{H}_{0}, \mathbf{p}\right]\right]\right| \Psi\right\rangle} .
$$

The adiabatic correction is defined as:

$$
\begin{aligned}
\delta E_{a d}= & -\frac{1}{M}\left\langle\Psi\left|\nabla_{\mathbf{R}}^{2}\right| \Psi\right\rangle-\frac{1}{4 M}\left\langle\Psi\left|\nabla_{\mathbf{r}}^{2}\right| \Psi\right\rangle= \\
& -\frac{1}{M}\left\langle\Psi\left|\left(\nabla_{\mathbf{R}} \mathrm{V}\right)\left(\mathrm{E}_{0}-\mathrm{H}_{0}\right)^{-1}\left(\mathrm{E}_{0}-\mathrm{H}_{0}\right)^{-1}\left(\nabla_{\mathbf{R}} \mathrm{V}\right)\right| \Psi\right\rangle-\frac{1}{4 M}\left\langle\Psi\left|\nabla_{\mathbf{r}}^{2}\right| \Psi\right\rangle
\end{aligned}
$$

where $\mathrm{E}_{0}$ is the non-relativistic ground-state energy. 
Let $n$ denote the number of electrons in the system. The wave function of a systems bound state can be written as:

$$
|\Psi\rangle=\sum_{i=1}^{n} c_{i}\left|\varphi_{i}\right\rangle
$$

where $c_{i}$ are linear expansion coefficients and $\varphi_{i}$ are basis functions.

We use the identity operator, I, expressed in terms of a non-ortogonal basis set.

This operator has the following spectral representation:

$$
\mathrm{I}^{p}=\sum_{a, b} c_{a}^{p}\left|\varphi_{a}^{p}\right\rangle\left\langle c_{b}^{p} \varphi_{b}^{p}\left|=\sum_{a, b}\right| \varphi_{a}^{p}\right\rangle c_{a}^{p} c_{b}^{p^{\dagger}}\left\langle\varphi_{b}^{p}\right| \quad \text { - for atoms }
$$

where $\varphi_{b}^{p}$ are basis function which are used to expand wave functions of states with the $p$ symmetry.

$$
\mathrm{I}=\sum_{a, b}\left|\varphi_{a}^{\Sigma}\right\rangle c_{a}^{\Sigma} c_{b}^{\Sigma^{\dagger}}\left\langle\varphi_{b}^{\Sigma}\left|+\sum_{a, b}\right| \varphi_{a}^{\Pi}\right\rangle c_{a}^{\Pi} c_{b}^{\Pi^{\dagger}}\left\langle\varphi_{b}^{\Pi}\right| \quad \text { - for molecules }
$$

where $\varphi_{a}^{\Sigma}$ and $\varphi_{a}^{\Pi}$ are sets of basis functions with the $\Sigma$ and $\Pi$ symmetries, respectively.

\section{Basis functions}

The all-electron explicitly correlated Gaussian functions with shifted centers (ECGs) used to expand the spatial part of the wave function for the ground state have the following form:

$$
\varphi_{k}^{(0)}=\exp \left[-\left(\mathbf{r}-\mathbf{s}_{k}\right)^{T} \underline{\mathbf{A}}_{k}\left(\mathbf{r}-\mathbf{s}_{k}\right)\right],
$$

for molecules and a similar form for atoms but without the shifts. In the above equation

- $\underline{\mathbf{A}}_{k}$ is a real $3 n \times 3 n$ symmetric positive-definite matrix of variational exponential parameters,

- $\underline{\mathbf{A}}_{k}=\mathbf{A}_{k} \otimes \mathbf{I}_{3}$, where $\mathbf{I}_{3}$ is the $3 \times 3$ unit matrix,

- $\mathbf{A}_{k}=\mathbf{L}_{k} \mathbf{L}_{k}{ }^{T}$, where $\mathbf{L}_{k}$ is an $n \times n$, rank $n$, lower triangular matrix,

- $\mathbf{s}_{k}$ is a $3 n$ vector of the coordinates of the Gaussian centers (shifts).

Gaussian function $\varphi_{k}^{(0)}$ are used to construct (by applying to them the $\nabla_{\mathbf{r}}$ operator) new basis functions $\varphi_{k}^{(x)}, \phi_{k}^{(y)}$, and $\varphi_{k}^{(z)}$ which are used to expand the special parts of wave functions of excited states. Those new basis functions have following form:

$$
\begin{aligned}
& \varphi_{k}^{(z)}=\left(z_{m_{k}}-s_{m_{k}}\right) \exp \left[-\left(\mathbf{r}-\mathbf{s}_{k}\right)^{T} \underline{\mathbf{A}}_{k}(\mathbf{r}-\mathbf{s}, k)\right], \\
& \varphi_{k}^{(x)}=\left(x_{m_{k}}\right) \exp \left[-\left(\mathbf{r}-\mathbf{s}_{k}\right)^{T} \underline{\mathbf{A}}_{k}\left(\mathbf{r}-\mathbf{s}_{k}\right)\right], \\
& \varphi_{k}^{(y)}=\left(y_{m_{k}}\right) \exp \left[-\left(\mathbf{r}-\mathbf{s}_{k}\right)^{T} \underline{\mathbf{A}}_{k}\left(\mathbf{r}-\mathbf{s}_{k}\right)\right] .
\end{aligned}
$$

In the calculation of the wave function expanded in terms of ECGs each Gaussian function is transformed with the appropriate permutational symmetry operator which is a sum of operators permuting the electron labels multiplied by appropriate linear coefficient. 


\section{Numerical tests}

The numerical results obtained using the algorithm described above are presented for the hydrogen atom in Table 1 and for the helium atom in Table 2. The results agree to within 2-4 significant digits with the literature data for all tested systems (the $\mathrm{H}, \mathrm{He}$, and $\mathrm{Li}$ atoms and the $\mathrm{H}_{2}, \mathrm{H}_{2}^{+}$ molecules).

\begin{tabular}{lclllll}
\hline \hline$N$ & $\ln k_{0}$ & $\mathrm{E}_{n r}$ & & \multicolumn{2}{l}{$\ln k_{0}$} \\
\hline 30 & 2.98198 & -0.49999999999021074 & & {$[3]$} & 2.811769883 \\
35 & 2.98342 & -0.49999999999996865 & & {$[4]$} & 2.984128555765498 \\
40 & 2.98369 & -0.49999999999999554 & & {$[5]$} & 2.9841285557654976107 \\
45 & 2.98400 & -0.49999999999999861 & & {$[2]$} & 2.984003 \\
50 & 2.98410 & -0.49999999999999874 & & \\
\cline { 1 - 2 }$\infty$ & & -0.5 & & \\
\hline \hline
\end{tabular}

Table 1: Hydrogen atom. Values of the Bethe logarithm, $\ln k_{0}$, and the non-relativistic energy, $\mathrm{E}_{n r}$, obtained in the basis set of $N$ ECGs functions.

\begin{tabular}{cccclll}
\hline \hline$N$ & $\mathrm{E}_{\mathrm{nr}}$ & $\ln k_{0}$ & & \multicolumn{2}{l}{$\ln k_{0}$} \\
\cline { 1 - 3 } \cline { 5 - 6 } 200 & -2.90372437193343912 & 4.408 & & {$[3]$} & $4.3740(4)$ \\
400 & -2.90372437685818471 & 4.397 & & {$[6]$} & $4.3701579(5)$ \\
600 & -2.90372437699588535 & 4.389 & & {$[8]$} & $4.370160218(3)$ \\
800 & -2.90372437702652771 & 4.384 & & {$[5]$} & $4.37016022306(2)$ \\
1000 & -2.90372437703109238 & 4.384 & & \\
\hline$[6]$ & -2.90372437703411959 & & & \\
\hline \hline
\end{tabular}

Table 2: Helium atom. Values of the Bethe $\operatorname{logarithm}, \ln k_{0}$, and the non-relativistic energy, $\mathrm{E}_{n r}$, obtained in the basis set of $N$ ECGs functions.

\section{Acknowledgments}

This work was supported by the National Science Center under Grant No. 2013/10/E/ST4/00033. Authors would like to express their gratitude to L. Adamowicz for helpful discussions.

\section{References}

[1] C. Schwartz, J.J. Tiemann, New calculation of the numerical value of the Lamb shift, Phys. Ann. 6, 178-187 (1959).

[2] M. Stanke, D. Kedziera, L. Adamowicz, Selection of a Gaussian basis set for calculating the Bethe logarithm for the ground state of the hydrogen atom, Mol. Phys. 11, 1062-1068 (2013).

[3] C. Schwartz, Lamb Shift in the Helium Atom, Phys. Rev 123, 1700 (1961). 
[4] G.W.F. Drake, R.A. Swaison, Bethe logarithms for hydrogen up to $n=20$, and approximations for two-electron atoms, Phys. Rev. A 43, 1243 (1990).

[5] V.I. Korobov, Calculation of the nonrelativistic Bethe logarithm in the velocity gauge, Phys. Rev. A 85, 042512 (2012).

[6] V.I. Korobov, S.V. Korobov, Bethe logarithm for the $1^{1} S$ and $2^{1} S$ states of helium, Phys. Rev. A 59, 3394 (1999).

[7] P.K. Kabir, E.E. Salpeter, Radiative corrections to the ground-state energy of the helium atom, Phys. Rev. 108, 1256 (1957).

[8] G.W.F. Drake, S.P. Goldman, Bethe logarithms for $\mathrm{Ps}^{-}, \mathrm{H}^{-}$, and heliumlike atoms, Can. J. Phys. 77, 835 (1999). 